\title{
Infectious Disease and Boxing
}

\author{
Osric S. King, $\mathrm{MD}^{\mathrm{a}, \mathrm{b}, \text { * }}$
}

\section{KEYWORDS}

- Boxing illnesses • Boxing respiratory diseases

- Boxing airborne infections • Boxing dermatologic infections

There are no unique boxing diseases but certain factors contributing to the spread of illnesses apply strongly to the boxer, coach, and the training facility. Like many athletes, boxers spend long hours preparing alone as well as with other boxers and coaches. The very nature of the exercise, surrounding environment, and final competition can increase the likelihood of infection through airborne, contact, and bloodborne routes of transmission. In addition, boxing is one of the unique sports in which the audience can potentially become contaminated and exposed to certain diseases. Fortunately, there have been no documented disease outbreaks associated with the sport. Also, the gross underreporting of boxing illnesses in the literature makes discussions of these situations more speculative than evidence-based. This article includes evidence from other sports such as running, wrestling, and martial arts to help elucidate the pathophysiologic elements that could be identified in boxers.

\section{IMMUNE SYSTEM PHYSIOLOGY}

Often, before an important fight a boxer is isolated from his usual environment in an attempt to enhance his focus by minimizing distractions. The additional benefit of the seclusion is that it minimizes exposure to potential infectious agents that could jeopardize his or her health before an important fight. Vigorous physical exertion produces alterations in the body's ability to fight infection. ${ }^{1}$ The circulatory system stimulates the changes in the immune system on several levels affecting the skin cells, upper respiratory tract mucosa, lung, peritoneal cavity, and muscles. Exerciseinduced stress affects the natural killer cells, neutrophils, and macrophages. Changes occur by influencing stress hormones, cytokine concentration, body temperature, blood flow, and hydration. The result is immune dysfunction lasting 3 to 72 hours. This dysfunction provides organisms a window of opportunity to increase the risk of subclinical and clinical infection. ${ }^{1,2}$ The overall effect appears to be modified by the degree of stress. ${ }^{3}$ There is an increased risk associated with heavy training, whereas moderate training has been associated with a reduction in upper respiratory tract

\footnotetext{
a Hospital for Special Surgery, 535 East 70th Street New York, NY 10021, USA

b New York State Athletic Commission

* Corresponding author. Hospital for Special Surgery, 535 East 70th Street, New York, NY 10021.

E-mail address: kingo@hss.edu
} 
infection (URTI) incidence. The risk of infection can also be amplified by the additional factors related to severe mental stress, sleep deprivation, and weight loss when a fighter has to make the necessary weight for an event. ${ }^{4}$

Hydration is a critical element for the body's immune system and there is evidence to suggest that dehydration can predispose an individual to developing a fever. ${ }^{5}$ In addition to being in optimal physical and mental condition, a boxer must make the weight stipulated by his division for the event. Because fat and muscle cannot be reduced quickly, dehydration becomes the main tool for losing a few pounds. The process can occur over a few months, weeks, days and, unfortunately, hours. Steam baths, plastic suits, laxatives, vomiting, and expectoration are used individually or in combination to achieve the desired weight loss. This activity can produce a potentially dangerous situation in regard to the boxer's susceptibility to infection. The mucosal layer of the oral pharynx is one of the first lines of protection from an invading organism. Exposure from the acidic contents of the stomach that occurs during induced vomiting directly compromises this barrier. In addition, diminishing the volume of saliva will also deplete the quantity of immunoglobulin A available to protect the oral and respiratory tract. Further volume depletion by the other methods can increase the heart rate and induce stress during a time when the boxer is relatively inactive. This stress can influence the body's ability to fight infection.

Diet and nutrition can play a role in counteracting some of the detrimental effects of exercise on the immune system. Water is the life blood of an active boxer during training and competition. Water is essential for providing the necessary hydration during exercise. Unfortunately, it contains none of the calories, proteins, or electrolytes necessary to enhance immune system function. Ingestion of carbohydrate beverages during intense and prolonged exercise seems effective in mitigating exercise-induced immune suppression. ${ }^{6}$ In addition, protein intake can increase insulin levels, optimize glycogen resynthesis, and enhance protein production necessary to fight infection. ${ }^{7}$ The use of some supplements such as quercetin also have the potential to lessen the magnitude of exercise-induced perturbations in immune function and reduce the risk of URTIs. ${ }^{8}$ Quercetin is found in onions, blueberries, curly kale, hot peppers, tea, and broccoli. Not only is quercetin a powerful antioxidant but it seems to also exert antipathogenic activity against a variety of viruses and bacteria. Other supplements such as vitamins $E$ and $C$ have not shown meaningful effects on exercise-induced inflammation, muscle damage, increases in plasma cytokines, and immune perturbations. . $^{9,10}$

In total, the physiologic effects of training seem to be more of a benefit then detriment because most conditioned athletes do not appear to experience any infectious diseases at a greater prevalence than the general population.

\section{ENVIRONMENT}

Intense training produces blood, sweat, and tears, which are among the nicer things found in a boxing gym. In studies, biochemical markers for blood, mucous, saliva, sweat, feces, and urine were detected on $36 \%$ of the surfaces in a daycare center. ${ }^{11}$ It is conceivable that in a gym wherein hygiene and hand washing are not emphasized, most surfaces will be contaminated. Locations such as the ring mat and ropes make contact with every external part of the human anatomy unprotected, or sponged through shirts and shorts soaked with a combination of water, blood, perspiration, urine, and saliva. Objects frequently struck such as speed or heavy bags can provide an effective means for making fungus, bacteria, and viruses airborne. To complicate matters, items such as boxing gloves, head gear, and protective gonad cups are infrequently sanitized with disinfectant and can act as infectious reservoirs. Fortunately, 
most boxers routinely shower after their workout, which theoretically can diminish the bacterial or viral load present on the epidermis especially if certain soaps are used..$^{12}$

\section{AIRBORNE INFECTIONS}

Similar to other individuals, the most likely illness a boxer will face is an URTI. Most of theses infections are from viruses but it is important to recognize bacterial infections and their potential complications. Also, supportive care for a viral infection should be selected carefully so as to avoid potentially dangerous reactions when symptom control medications are used during exercise activities.

The second most common illness seen in a United States physician's office is the common cold. ${ }^{13}$ The initial symptoms consist of sore throat, malaise, and low-grade fever. These symptoms may resolve in a few days and are followed by rhinorrhea, cough, and nasal congestion. The symptoms usually peak around day three or four and usually resolve by day seven. ${ }^{14}$ Rhinovirus is the most common source but the common cold can be caused by one of several viruses including coronavirus, respiratory syncytial virus, influenza virus, parainfluenza virus, and adenovirus. There are no effective antivirals to cure the common cold and few effective measures to prevent it. Antibiotics are inappropriately prescribed to improve cure rate and symptom persistence, and prevent secondary bacterial complications. Systematic reviews have shown that antibiotics are ineffective in controlling symptoms and have no role in the treatment of the common cold. ${ }^{15,16}$ Furthermore, antibiotics increase the risk of gastrointestinal effects, allergic reactions, and resistance of bacteria to antibiotics. Treatment of a cold should focus on controlling the symptoms. Nontraditional complementary and alternative therapies used for the common cold include Echinacea, vitamin $\mathrm{C}$, zinc, and humidified air and fluid intake. ${ }^{17}$ Two well-conducted studies and a Cochrane review showed no benefit from using Echinacea to treat or prevent the common cold. ${ }^{18-20}$ There is no evidence that vitamin $\mathrm{C}$ decreases symptom severity or duration when initiated after the onset of cold symptoms. However, when used prophylactically, an exercise group taking vitamin C had a $50 \%$ relative reduction in the risk of developing a cold. Studies with zinc show inconsistent viral growth inhibition and reduction of cold symptoms duration. ${ }^{21}$ The use of humidified air and fluid intake are considered benign and possibly beneficial for the relief of common cold symptoms. ${ }^{16}$ A Cochrane review showed some evidence that dextromethorphan provided a modest clinical benefit controlling coughs. Overall, however, there is a lack of evidence to determine the effectiveness of any over-the-counter product at reducing the frequency or severity of cough in children or adults. ${ }^{22}$ The American College of Chest Physicians guidelines do not recommend centrally acting cough suppressants (codeine or dextromethorphan) for cough secondary to URTI. ${ }^{23}$ For nasal congestion and rhinorrhea symptoms, antihistamines remain a popular therapy and the older first-generation products have shown positive results for certain end points. However, a Cochrane review concluded that antihistamines do not alleviate cold-related sneezing or nasal symptoms to a clinically significant degree, and do not affect subjective improvement in children or adults. ${ }^{24}$ Studies appear to support the short-term use of intranasal and oral decongestants as well as the use of topical ipratropium (Atrovent) for rhinorrhea. ${ }^{16}$ Extreme symptoms may warrant the use of some antihistamines, decongestants, dextromethorphan and, most importantly, rest. Antihistamines, dextromethorphan, and decongestants, even without exertion, can cause cardiac arrhythmias, blurred vision, hypertension, seizures, respiratory depression, irritability, confusion and irritability, and sedation. ${ }^{16}$ In a boxer, the use of these medications combined with rigorous training, let alone a fight, could be 
potentially fatal. There are also studies relating a risk of stroke to the over-the-counter cough and cold drugs. The mechanism seems related to hypertension or vasospasm or angiitis. ${ }^{25}$ Overall, the gain in taking cold symptom medication to train or perform more comfortably does not outweigh the potential risk from the side effects.

\section{Acute Sinusitis}

Acute sinusitis is defined as the inflammation of the mucosal lining of the paranasal sinuses lasting less than 4 weeks. Viral URTI is often the trigger for bacterial sinusitis, ${ }^{26}$ with about $0.5 \%$ of common colds becoming complicated by the development of acute sinusitis. ${ }^{27}$ Viral infection is the most common cause of acute sinusitis, and usually resolves in 7 to 10 days. Acute bacterial sinusitis is also self-limiting, with $75 \%$ of cases resolving without treatment within 1 month. ${ }^{28}$ The usual pathogens are Streptococcus pneumoniae and Haemophilus influenzae with occasional infection with Moraxella catarrhalis. The diagnosis is most commonly based on clinical history and physical findings. Laboratory tests can assist in chronic or complicated cases but imaging studies may not be useful because asymptomatic sinusitis is often reported on a boxer's magnetic resonance imaging (MRI) or computed tomography (CT) scans. The symptoms consist of nasal congestion, purulent nasal discharge, maxillary tooth discomfort, headaches, fever, and facial pain or pressure that is worse when leaning forward. Treatment is often sought when training is complicated by the inability to breathe through the nose. This situation helps to distinguish traumatic headache from sinus headache. Left untreated, some bacterial sinusitis may not spontaneously resolve and can have severe complications including intracranial and orbital infections. Superimposed facial trauma could potentially predispose or advance the disease. The use of cephalosporins and macrolides seem to be as effective as, and have fewer adverse effects than amoxicillin or amoxicillin-clavulanate. Intranasal corticosteroid sprays and decongestants may be effective in reducing symptoms. There do not appear to be any studies supporting the effectiveness of decongestants, antihistamines, saline nasal washes, and steam inhalation in reducing the duration of the illnesses. ${ }^{29}$ However, the latter two treatments offer the most benefit and the least risk of adverse effects.

\section{Pharyngitis}

When eating or swallowing food is painful, most boxers will seek medical attention. Although most sore throats have an infectious origin, fewer than $20 \%$ have a clear indication for antibiotic therapy. Bacterial pathogens include group C and group G Streptococcus, mixed anaerobes, Corynebacterium diphtheria, and several chlamydial species. The major common pathogen warranting treatment is Group A b-hemolytic streptococcus (GABHS), which accounts for $5 \%$ to $15 \%$ of cases and, infrequently, can be responsible for rheumatic fever, acute glomerulonephritis, and peritonsillar abscess. ${ }^{30}$ Pharyngeal swelling, tonsillar exudates, fever, and tender anterior cervical lymph nodes and a scarlet rash suggest GABHS infection. Laboratory analysis clarifies the decision to treat with antibiotics. Throat cultures remain the gold standard for confirming the diagnosis, with sensitivity approaching $97 \%$ and specificity ranging from $95 \%$ to $99 \% .^{31}$ Rapid antigen detection tests are almost as sensitive as throat cultures. These tests are readily available and easy to perform, with results available within minutes. ${ }^{32}$ When individuals fail to improve with antibiotic treatment, other origins of their symptoms may include mononucleosis and possibly gastroesophageal reflux. In boxers who are sexually active and present with fever, sore throat, dysuria, and characteristic greenish exudates, gonococcal pharyngitis should be considered. ${ }^{33} \mathrm{~A}$ rash following the administration of antibiotics can indicate a drug allergy 
or possible viral infection with mononucleosis. In addition to high fever, pharyngitis, and lymphadenopathy, the boxer with mononucleosis may classically present with extreme fatigue (especially after the acute symptoms have resolved). Laboratory analysis is important in confirming the disease and may show atypical lymphocytes, elevated mononucleosis titers, and elevated liver function tests. Testing during mild symptoms or early in the episode may yield a false positive. A major risk for boxers is the possibility of splenomegaly and subsequent splenic rupture with activity. The incidence of splenic rupture is 1 to 2 cases per $1000,{ }^{34}$ and occurs spontaneously in half of the cases between the fourth and 21 st days of symptomatic illness. ${ }^{35}$ The major concern is that direct thoracic and abdominal blows are expected in boxing and could be fatal in the presence of splenomegaly. Ultrasound evaluation of the spleen size is of some value but $7 \%$ of athletes' baseline spleen size meets the current criteria for splenomegaly. ${ }^{36}$ Another concern for mononucleosis is its long incubation period and potential for asymptomatic spread. Contamination occurs through contact with saliva. This contact may occur directly through the sharing of water bottles or possibly indirectly because saliva may be present on gloves, ropes, or other surfaces.

\section{Community-Acquired Pneumonia}

The term community-acquired pneumonia (CAP) is used when the patient has not been hospitalized or in a long-term facility for at least 14 days before the onset of symptoms. The symptoms include cough, fever, chills, fatigue, dyspnea, rigors, and pleuritic chest pain. The expected musculoskeletal discomfort from training could make the early detection of pneumonia difficult in a boxer. Origins such as Legionella may also produce gastrointestinal symptoms. Typical pneumonia is caused by Streptococcus pneumoniae and is found in very young or older patients. Atypical pneumonias are found most often in young adults and are usually caused by influenza virus, Mycoplasma, Legionella, Chlamydia, and adenovirus. ${ }^{37}$ Chest radiography is the gold standard in diagnosing pneumonia but may show as falsely negative early in the disease. The validity of common laboratory tests such as leukocyte count, sputum stain, and blood cultures have been questioned after low positive culture rates were found. ${ }^{38}$ Antibiotics that provide coverage against the most common organisms known to cause CAP should be used for treatment.

\section{Bronchitis}

Acute bronchitis is inflammation of the bronchial mucus membranes. Acute bronchitis should be suspected when a boxer presents coughing with or without sputum lasting up to 3 weeks with evidence of concurrent upper airway infection. The most common cause is viral infections. Less than $10 \%$ have bacterial origin. The most common viruses are influenza $A$ and $B$, adenovirus, rhinovirus, parainfluenza virus, coronavirus, and respiratory syncytial virus. The bacteria found in acute bronchitis are Bordetella pertussis, Mycoplasma pneumoniae and Chlamydia pneumoniae. ${ }^{39}$ Despite improvements in testing and technology, diagnosis of bronchitis is mostly clinical because no routinely performed procedures diagnose acute bronchitis. Chest radiography should be reserved for use in patients whose physical examination suggest pneumonia or heart failure, and in patients who would be at high risk if the diagnosis were delayed. ${ }^{40}$ The recommended therapy is symptomatic care if the clinical diagnosis of acute bronchitis is established. Treatment should focus on preventing or controlling the cough (antitussive therapy) or on making the cough more effective (protussive therapy). Terbutaline, amiloride, and hypertonic saline aerosols have proved successful in protussive treatment of coughs from various causes. Studies with guaifenesin were inconclusive because the dose evaluated was less than what is usually prescribed. ${ }^{41}$ 
Antitussive selection is based on the cause of the cough. An antihistamine would be used to treat cough associated with allergic rhinitis, a decongestant or an antihistamine would be selected for cough associated with postnasal drainage, and a bronchodilator would be appropriate for cough associated with asthma- and nonasthma-related conditions. ${ }^{42}$ Nonspecific antitussives such as hydrocodone, dextromethorphan, and codeine simply suppress cough and their side effects should be considered when a boxer is training.

\section{Pericarditis}

In a boxer it is crucial to distinguish between cardiac chest pain and training-related musculoskeletal soreness. Pericarditis is the inflammation of the pericardium, the sac surrounding the heart. Pericarditis is most often secondary to a viral infection but it may also be caused by other diseases, drugs, invasive cardiothoracic procedures, and chest trauma. ${ }^{43}$ Symptoms may be mild with no significant discomfort or they might present with sudden onset of severe substernal chest pain. Viral origins include coxsackievirus $A$ and $B$, hepatitis viruses, human immunodeficiency virus (HIV), influenza, measles, mumps, and Varicella. Bacterial causes include gram-positive and gram-negative organisms and, rarely, Mycobacterium tuberculosis. Fungal origins are seen in immunocompromised patients: Blastomyces dermatidis, Candida, and Histoplasma capsulatum. A boxer may present with a history of fever, malaise, and myalgias. The cardinal features of acute pericarditis are chest pain, which is left-sided and may radiate to the trapezius, neck, arms, or jaws. With this pain distribution in mind, it is understandable that the symptoms are easily misinterpreted; this is especially true after extensive training. The detection of pericardial friction rub on auscultation has a high specificity but a low sensitivity that varies with the frequency of auscultation. ${ }^{44}$ The electrocardiogram is abnormal in $90 \%$ of pericarditis cases. ${ }^{45}$ Laboratory analysis may show evidence of inflammation with elevated C-reactive protein, erythrocyte sedimentation rate and leukocyte count. Markers of myocardial injury such as the MB isoenzyme of creatine kinase and cardiac troponins are also elevated. Echocardiography may be normal, CT and MRI are useful if the initial workup for pericarditis is inconclusive. ${ }^{46}$ The goal of treatment is to relieve pain and prevent complications such as recurrence, tamponade and chronic restrictive pericarditis. Whether to manage the patient as an outpatient or inpatient will depend on the level of complications and severity.

\section{Myocarditis}

Another source of cardiac chest pain in a boxer is myocarditis, which is the inflammation of the cardiac muscle. Myocarditis is most commonly caused by viruses such as coxsackievirus B, adenovirus, hepatitis $\mathrm{C}$ virus, cytomegalovirus, echovirus, influenza virus, and Epstein-Barr virus. The boxer may be asymptomatic, giving a history of a preceding URTI, or he might present with symptoms of chest pain or heart failure. The physical examination may reveal a muffled first heart sound along with a third heart sound and a murmur of mitral regurgitation. Lower extremity edema along with pulmonary crackles from fluid overload may indicate the severity of the condition. Routine blood tests are generally normal but creatine kinase-MB and troponin I may be elevated. Electrocardiographic findings are transient and may show nonspecific ST-T wave abnormalities. Chest radiographs may show pulmonary congestion and cardiomegaly. Echocardiograms are useful and may reveal global decreased ventricle dysfunction. Treatment is generally supportive for viral causes with the emphasis on treating heart failure and potential arrhythmias in serious cases. ${ }^{47}$ Following the acute illness, myocarditis as well as pericarditis can cause $11 \%$ of sudden and unexpected cardiac death during or after physical exercise. ${ }^{48}$ Surprisingly, some individuals are 
asymptomatic. ${ }^{49}$ Because of this danger, Bethesda Conference recommends that athletes who have probable or definitive evidence of myocarditis should be withdrawn from all competitive sports and undergo a prudent convalescent period of about 6 months.

\section{CONTACT INFECTIONS}

Skin infections are often the result of a break in the integrity of the skin. The organism can be fungi, virus, or bacteria. Most boxers will experience facial lacerations during the course of their career. What contaminates the wound depends on two factors: the organism colonizing the skin or glove before impact and what was used to control the bleeding that followed. Ideally, sterile gauze applied by an experienced "cut man" using a swab will be applied to the lesion. It is likely that the "corner towel" previously used to absorb the boxer's perspiration and possibly wipe water from the mat is implemented to achieve hemostasis. With this in mind, there is enormous potential for infections.

\section{Fungus}

In boxing, the skin's mechanisms of protection fails because of repeated trauma, irritation, and maceration during the course of training. In addition, skin occlusion with nonporous materials, seen with some protective head and gonad gear, interfere with the skin's barrier function. This interference occurs by increasing local temperature and hydration. ${ }^{50}$ In wrestlers, fungal infections, termed tinea corporis gladitorum, can be seen in up to $75 \%$ of participants. The transmission is primarily through skin-toskin contact. Lesions are typically found on the head, neck, and arms. ${ }^{51}$ Although there is different, and relatively less, contact made in boxing compared with wrestling, the potential for contamination exists. Microsporum, Trichophyton, and Epidermophyton species are the most common fungal pathogens in skin infections. These pathogens are categorized as dermatophytes or tinea because they are fungi that require keratin (found in skin, hair, and nails) for growth. Superficial skin infections are caused less frequently by nondermatophyte fungi such as Malassezia furfur in tinea/pityriasis versicolor, and Candida species. Transmission can occur by direct contact or from exposure to desquamated cells. Inoculation occurs through breaks in the skin. Germination occurs following the invasion of the superficial skin layers. The physical examination typically reveals an inflammatory response characterized by a greater degree of redness and scaling at the edge of the lesion or occasionally by blister formation. Potassium hydroxide $(\mathrm{KOH})$ microscopy aids in visualizing hyphae and confirming the diagnosis of dermatophyte infection. Other diagnostic modalities include Wood's lamp examination, fungal culture, and skin or nail biopsy. ${ }^{52}$ Topical treatment is usually adequate for most tinea except tinea capitis, tinea barbae, tinea unguium and, rarely, tinea corporis.

\section{Virus}

Sexual activity, skin-to-skin contact, and the contaminated surfaces of a gym are high-risk routes of viral infection. Molluscum contagiosum (MC), human papillomavirus (HPV), and herpes are the most likely viruses to infect the skin of a boxer.

$M C$ infections occur frequently among children and also affect sexually active adults. ${ }^{53} \mathrm{MC}$ may also serve as a cutaneous marker of severe immunodeficiency and sometimes is the first indication of HIV infection. ${ }^{54} \mathrm{MC}$ is a double-stranded DNA virus in the Poxviridae family. $M C$ is spread through fomite or skin-to-skin contact, and abrasions in the epidermis are thought to facilitate transmission. ${ }^{55}$ The 
typical appearance is an asymptomatic, firm, smooth, round papule with central umbilication, typically found on the extremities, trunk, and face. In sexually transmitted cases they can be found on the lower abdomen and in the genital region. Spontaneous resolution of the lesions occurs but eradication is possible through mechanical (curettage, laser, cryotherapy), chemical (trichloroacetic acid, tretinoin), or immunologic (imiquimod) means.

Human papillomavirus causes warts that can occur almost anywhere on the body. After initial infection by direct contact from skin or a contaminated surface, warts are frequently spread by autoinoculation from scratching, shaving, or skin trauma. The appearance may vary from an irregularly surfaced domed lesion (common warts) to a filiform projection seen on the face, to flat plantar warts on the plantar surface of feet. Plantar warts tend to be painful because they become callused and grow into the foot instead of rising above the surface. Treatment options for warts include mechanical destruction and adjustment of the patient's immune system through medications.

Skin infection with herpes simplex virus (HSV) poses a huge risk to boxers. HSV affects more than one-third of the world's population and is responsible for a wide array of symptoms, and may occasionally lead to death. There are two separate types, labeled HSV-1 and HSV-2. Each type has affinities for different body sites. HSV-1 causes $90 \%$ of oral lesions and HSV-2 causes $90 \%$ of genital lesions. Both viruses enter the host through abraded skin or intact mucous membranes. Epithelial cells are the initial targets. Once infected these cells die, releasing clear fluid intradermally to form vesicles. Retrograde transport through adjacent neural tissue to sensory ganglia leads to lifelong latent infection. ${ }^{56}$ The initial infection is usually due to skinto-skin contact. Transmission can potentially occur from inanimate objects, as in the case of an English boxer whose glove, contaminated with smallpox, was reported to infect his Norwegian opponent in $1948 .{ }^{57} \mathrm{HSV}$ has been reported to survive on plastic spa surfaces at temperatures around $100^{\circ} \mathrm{F}\left(38^{\circ} \mathrm{C}\right)$ for up to 4.5 minutes. ${ }^{58}$ To complicate matters further, asymptomatic shedding of viral particles happens frequently, making infection identification and prevention based on visualizing lesions extremely ineffective. Reactivation of the virus causes recurrent symptoms and is triggered by local or systemic stimuli such as ultraviolet light (UV), trauma, fever, and immunodeficiency. During training, a boxer may be exposed to all of these factors individually or in combination. Latent HSV-1 may reactivate in boxers during exposure to UV light from the sun that may occur during running or "road work." 59 Trauma from punches to the face, with and without head gear, during sparring sessions could also contribute to developing HSV-1 lesions. Physical and mental stress can induce immune suppression and has been shown to increase HSV-associated pathogenicity. Once HSV is located in a boxer's skin, the incubation period ranges from a few days to several weeks. First a prodrome characterized by burning, stinging, itching, or pain in the area, eventually develops into a skin lesion. Very early lesions have a nonspecific clinical appearance, usually as well-defined erythematous papules. As the individual lesions resolve, a crust develops on top. Systemic symptoms are not unusual, especially in cases of primary HSV infection. Athletes may complain of fever, sore throat, malaise, myalgias, arthralgias, and swollen lymph nodes. The recurrent symptoms around the face are usually self-limiting without treatment. The potential for eye and central nervous system involvement is the greatest risk for boxers. Involvement of the eye (including conjunctivitis, blepharitis, and keratitis) has been well documented in wrestlers. ${ }^{60}$ There is also a documented case of a rugby player developing HSVrelated meningitis and sacral ganglionitis with perineal and lower extremity paresthesias. ${ }^{61}$ Acyclovir, famciclovir, and valacyclovir are the main antivirals used for treating 
HSV infections. There are financial and convenience factors involved in deciding which medication to use. Overall, treatment is valuable because it lessens the pain, degree of viral shedding, constitutional symptoms, and lesion healing time.

\section{Bacterial}

Second to HIV, there is arguably no infectious organism that has received as much recent publicity as methicillin-resistant Staphylococcus aureus (MRSA). MRSA infections have been a complication in hospital settings for over 40 years. ${ }^{62}$ Hospitalization with some degree of immunosuppression and antibiotic use were previously key predisposing factors. In the past few years its presence outside health care centers in immunocompetent individuals with no history of antibiotic use has made MRSA a media favorite. The presence of MRSA in training facilities and in some popular athletes has forced the entire sports community to look closely at current hygienic practices. High school, collegiate, and professional sports have published case reports. Although contact sports such as football, wrestling, and rugby have the most documented cases, infections in athletes participating in basketball, fencing, and weightlifting have also been reported. ${ }^{63}$ At the time of writing there were no cases of MRSA in a boxer and only one incidence of a mixed martial arts (MMA) fighter competing with an MRSA infection, which was diagnosed after the event. Thinking he was suffering the effects of rapid weight loss, the athlete ignored signs of fever and vomiting before the fight. ${ }^{64}$ Supersaturation of the stratum corneum (the first outer layer of skin) by sweating combined with abrasions and cuts allow the entrance of the microorganism through the epidermis. There is also some link to environmental surfaces, whirlpools, taping gel, and towels. ${ }^{65,66}$ There have been no case reports of MRSA in boxing, possibly reflecting underreporting because the predisposing factors exist in abundance. The lesions can present in the form of impetigo, folliculitis, or furunculosis. There are also some reports of periorbital cellulitis in the nonathletic population. Impetigo lesions are characterized by well-defined erythematous papules and plaques with honey-colored crust, with or without pustules. These lesions can also present with discrete clear fluid-filled vesicles that coalesce into larger bullae. The infection can occur with skin-to-skin contact and can be spread further by scratching. Culture confirms the diagnosis. Impetigo can be treated with warm soaks and topical antibiotics but extensive disease necessitates oral antibiotics. Folliculitis is hair follicles that become inflamed by physical injury, chemical irritation, or infection. The hair shaft is frequently be seen in the center of the pustule. Furuncles and carbuncles can occur as follicular infections or impetigo progress deeper. Such a lesion is commonly known as an abscess or boil, and consists of a tender, erythematous, firm, or fluctuant mass of walled-off purulent material. Constitutional symptoms including fever and malaise are common. Treatment often consists of incision and drainage accompanied by oral or, in severe cases, parenteral antibiotics. For most of the bacterial skin lesions, return to sporting activity is after 48 to 72 hours of systemic antibiotics and no new or active draining lesions may be evident at the time of activity. ${ }^{67}$

\section{BLOODBORNE INFECTION}

$\mathrm{HIV}$, hepatitis $\mathrm{B}$ virus (HBV), and hepatitis $\mathrm{C}$ virus $(\mathrm{HCV})$ are the most common bloodborne pathogens. Boxers are at high risk of spreading or contracting an infection because bleeding occurs frequently during competition. The mechanism of transmission is similar to the general population, and can include using a contaminated needle as well as direct contact from an infected boxer to the opponent's damaged skin or 
a mucous membrane. In boxing there are several precautions in place to limit the spread of these diseases. The professional boxing commissions in most US states require mandatory periodic testing for these diseases before allowing professional boxers to compete. The amateur boxing supervising authority (USA Boxing) has taken measures to limit excessive bleeding during competition but does not test for infections.

\section{Human Immunodeficiency Virus}

HIV replicates in human cells expressing the T4 (CD4) antigen and causes suppression of the immune system. The individual with HIV has weakened defenses against invading organisms and is susceptible to opportunistic infections which, left untreated, ultimately lead to death. HIV created a huge dilemma for boxing and the other sports in which bleeding can occur. After concerned physicians advocated safer protocols, mandatory testing was initiated early followed by recommendations to stop a bout until bleeding was controlled. ${ }^{68,69}$ Safety measures such as universal precautions and mandatory testing were incorporated quickly by most boxing commissions. In boxing, guidelines for excessive bleeding were easy to implement because controlling bleeding is always an important objective for strategic and psychological reasons. Excessive blood cannot only impair vision but distract a boxer if he is constantly trying to wipe it away. Some boxers will also display diminished skills at the sight of their own blood. Overall, the two preventative strategies of testing and bleeding control seem effective in minimizing the incidence of boxing-related transmission of HIV. There is no evidence that HIV can be transmitted through saliva, sweat, or tears, ${ }^{68}$ so there is no danger associated with normal body contact such as touching and sharing sports equipment or towels. Also, contaminated surfaces such as ring mats, toilet seats, and hot tubs do not pose risks. According to the US Centers for Disease Control and Prevention (CDC), the risk of transmission of HIV during sports is small and there are no documented cases of HIV contamination during sports. ${ }^{70}$ Evidence exists suggesting that the baseline risk of HIV may be higher in competitive athletes. This evidence seems more related to "risky" lifestyle behavior including increased alcohol consumption, inconsistent contraceptive use, and multiple sex partners than the inherent risks of the sport. ${ }^{71}$ Intramuscular injection of anabolic steroids and other performance-enhancing drugs constitutes another HIV risk behavior associated with athletes. The case of a body builder with acquired immune deficiency syndrome (AIDS) suggests that like in the general population, contaminated needles are also a source of HIV infection. ${ }^{72}$ There is a report of HIV seroconversion as a result of bleeding during a soccer match in Italy. ${ }^{73}$ After further investigation, transmission through nonsports activity could not be ruled out because the man involved also worked in a drug dependency rehabilitation program. There are also some case reports of HIV transmission during bloody street fights. ${ }^{74,75}$ These events should not be compared with boxing, because they lack the rules and regulations that minimize the dangers and limit morbidity and mortality. Evidence of HIV infection can be found by testing for the antibodies (enzyme-linked immunosorbent assay [ELISA] and Western blot test). Unfortunately, the tests do not become positive until 3 to 4 weeks (sometimes even months) after infection. Quantifying HIV RNA level (viral load) by polymerase chain reaction (PCR), which is $95 \%$ to $98 \%$ sensitive for $\mathrm{HIV}$, becomes positive within 11 days of infection. ${ }^{76}$ At present the ELISA and Western blot tests are the accepted screening tests for boxing. A possible way to strengthen the current protocols may be by mandating viral load testing and including amateur as well as professional boxers. Justifying a change in tests may not be easy because antibody testing is the accepted standard and seems to be working well. There is no denying that that in 
amateur bouts the chance of HIV transmission through lacerations is less, but not impossible, with the use of headgear. However, nose bleeds are very common and could increase the risk of transmission. Testing amateur boxers may not be cost effective but it would make the disease-screening process more thorough. There is some opinion that the physical demands of the sport would limit participation by athletes infected with HIV. ${ }^{77}$ The opposite may be true, with the exertion from boxing training potentially being beneficial for an individual with HIV. ${ }^{78}$ Finally, with the current regimen of medications that are able to minimize the viral loads, a boxer with HIV may be able to compete without any obvious limitations and it may not be detectable with the current screening methods. There is a famous case of a boxer diagnosed with HIV who continues to fight professionally. His case is unusual because his initial test was reported positive but subsequent tests have all been negative. It is uncertain whether he was the victim of a false positive, or for whatever reasons, his virus is presently undetectable. At present most professional boxing commissions are not willing to take a chance and allow him to continue competing in their states.

\section{Hepatitis B Virus}

The most common mode of HBV transmission is via intimate sexual contact. When considering direct contact with blood, it is more likely than HIV to be transmitted during boxing because it is present in higher concentrations in the blood and more stable in the environment outside of the body and laboratory. HBV outbreaks in sports are not uncommon and contact with open wounds of HBV carriers is thought to be the precipitating factor. Five of 10 members of a Japanese high school sumo wrestling club contracted hepatitis during a single year. In 2000 an American football team reported that 11 of 65 athletes were found to have HBV over a 19-month period. Most recently, a study revealed 9 of 70 Turkish Olympic wrestlers to have occult HBV infection. The study suggested that sweat may be another way of transmitting HBV infections. At the time writing, two world championship fights were called off because one of the participants was found to have HBV. ${ }^{79,80}$ The presence of certain antigens detected by laboratory analysis can determine past, current, or chronic HBV infections (Box 1). ${ }^{81}$

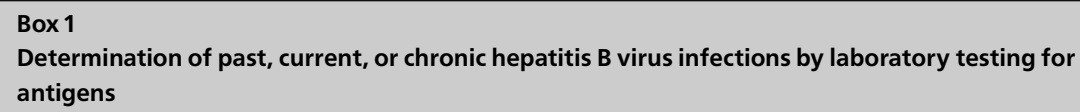

Hepatitis B surface antigen (HBsAg): Present in acute or chronic infection

Hepatitis $B$ surface antibody (anti-HBS): Marker of immunity acquired through natural HBV infection, vaccination or passive antibody

Hepatitis $B$ core antibody (anti-HBc):

IgM-indicates infection in the previous 6 months

IgG-indicates prior HBV infection that may have been cleared by the immune system or that may persist; positive $\mathrm{HBsAg}$ and anti-HBC

IgG-indicates persistent chronic HBV infection

Hepatitis Be antigen ( $\mathrm{HBeAg}$ ): Marker of infectivity that correlates with high viral replication Hepatitis Be antibody (anti-HBe): Correlates with low viral replication

HBV DNA: Correlates with active replication; useful in monitoring treatment responses 
A boxer with HBV may be asymptomatic or present with myalgia, urticaria, joint pain, nausea, anorexia, low-grade fever, or abdominal pain. Patients may be treated with immune modulators such as interferon or antivirals. No information is available at this time regarding whether boxers treated for HBV can safely return to competition. Many American-born children are vaccinated for HBV, which will help to minimize the prevalence of HBV in the future.

\section{Hepatitis C Virus}

$\mathrm{HCV}$ is the most common bloodborne infection, the leading cause of hepatocellular carcinoma, and the principal diagnosis among patients referred for liver transplantation. Acute HCV infection is rarely severe and usually asymptomatic. The virus is transmitted through exposure to infected blood and in the sports world most known transmissions have occurred through shared needles. One observed case involved the injection of anabolic androgenic steroids. ${ }^{82}$ Another report involved three amateur soccer players infected with HCV after sharing a syringe to inject vitamin complexes. ${ }^{83}$ There was a case in 2000 in which HCV was transmitted during a bloody fight between two family members. At the end of the fight they shared a handkerchief to dry their blood so it is uncertain when the inoculation occurred, during the fight or afterwards. The diagnosis is made by antibody (anti-HCV) testing, which is $99 \%$ sensitive and $99 \%$ specific. Anti-HCV determines whether a person was exposed but does not detect the presence of active infection. Because some individuals spontaneously clear the infection, the presence of HCV RNA (quantitatively or qualitatively) is used for confirmation. False-negative results for HCV antibody testing may occur in immunosuppressed persons.

\section{SUMMARY}

Boxers are at increased risks for certain infectious diseases because of certain training, environmental, and sociologic parameters. The intensive mental and physical stresses of the sport can alter the ability to fight off disease. The inappropriate use of medications for upper respiratory infections may do more harm than good. The training facility may also influence what fungi, viruses, and bacteria are present to cause infections. Like other sports, dangerous bloodborne diseases are acquired by risky behavior, particularly sexual, and sharing needles to inject performanceenhancing drugs. Precautions are necessary to detect and prevent the spread of illnesses to both participants and observers of boxing. There are treatment options available but the risks and side effects must be weighed against the potential benefits. For some diseases treatment does not guarantee a return to competitive sport.

\section{REFERENCES}

1. Nieman DC. Marathon training and immune function. Sports Med 2007;37(4-5): 412-5.

2. Kruger K, Mooren FC. T cell homing and exercise. Exerc Immunol Rev 2007;13: 37-54.

3. Nieman DC. Exercise and resistance to infection. Can J Physiol Pharmacol 1998; 76(5):573-80.

4. Nieman DC. Special feature for the olympics: effects of exercise on the immune system: exercise effects on systemic immunity. Immunol Cell Biol 2000;78(50): 496-501.

5. Morimoto A, Murahami N, Ono T, et al. Dehydration enhances endotoxin fever by increased production of endogenous pyrogen. Am J Physiol 1986;25(1 Pt2):R41-7. 
6. Walker GJ, Finlay $\mathrm{O}$, Griffiths $\mathrm{H}$, et al. Immunoendocrine response to cycling following injestion of caffeine and carbohydrate. Med Sci Sports Exerc 2007; 39(9):1554-60.

7. Kreider RB, Earnest CP, Lundberg J, et al. Effects of ingesting protein with various forms of carbohydrate following resistance-exercise on substrate availability and markers of anabolism, catabolism, and immunity. J Int Soc Sports Nutr 2007;4:18.

8. Nieman DC. Immunonutrition support for athletes. Nutr Rev 2008;66(6):310-20.

9. Nieman DC, Peters EM, Henson DA, et al. Influence of vitamin C supplementation on cytokine changes following an ultramarathon. J Interferon Cytokine Res 2000; 20:1029-35.

10. Peterson EW, Ostrowski K, Ibfel T, et al. Effect of vitamin supplementation on cytokine response and on muscle damage after strenuous exercise. Am J Physiol Cell Physiol 2002;280:C1570-5.

11. Reynolds KA, Watt PM, Boone SA, et al. Occurrence of bacteria and biochemical markers on public surfaces. Int J Environ Health Res 2005;15(3):225-34.

12. Kaiser AB, Kernodle DS, Barg NL, et al. Influence of preoperative showers on staphylococcal skin colonization: a comparative trial of antiseptic skin cleaners. Ann Thorac Surg 1988;45(1):35-8.

13. Woodwell DA, Cherry DK. National ambulatory medical care survey: 2002 summary. Adv Data 2004;346:1-44.

14. Heikkinen T, Jarvinen A. The common cold. Lancet 2003;361:51-9.

15. Arroll B, Kenealy T. Antibiotics for the common cold and acute purulent rhinitis. Cochrane Database Syst Rev 2005;(3):CD000247.

16. Fahey $T$, Stocks N, Thomas T. Systematic review of the treatment of upper respiratory tract infection. Arch Dis Child 1998;79:225-30.

17. Simasek M, Bladino D. Treatment of the common cold. Am Fam Physician 2007; 75(4):515-20.

18. Linde K, Barrett B, Wolkart K, et al. Echinacea for preventing and treating the common cold. Cochrane Database Syst Rev 2006;(1):CD000530.

19. Yale SH, Liu K. Echinacea purpurea therapy for the treatment of the common cold: a randomized double-blind, placebo-controlled clinical trial. Arch Intern Med 2004;164:1237-41.

20. Turner RB, Bauer R, Woelkart K, et al. An evaluation of Echinacea angustifolia in experimental rhinovirus infections. N Engl J Med 2005;353:341-8.

21. Marchall I. Zinc for the common cold. Cochrane Database Syst Rev 1999;(2):CD001364.

22. Schroeder K, Fahey T. Over-the-counter medications for acute cough in children and adults in ambulatory settings. Cochrane Database Syst Rev 2004;(4):CD001831.

23. Irwin RS, Baumann MH, Bolser DC, et al. American College of Chest Physicians. Diagnosis and management of cough executive summary: ACCP evidencebased clinical practice guidelines. Chest 2006;129(Suppl 1):1S-23S.

24. Sutter Al, Lemiengre M, Campbell H, et al. Antihistamines for the common cold. Cochrane Database Syst Rev 2003;(3):CD001267.

25. Cantu C, Arauz A, Bonilla L, et al. Stroke associated with symptathomimetics contained in over-the-counter cough and cold drugs. Stroke 2003;34:1667-72.

26. Hentry DC, Moller DJ, Adelglass J, et al. Comparison of sparfloxacin and clarithromycin in the treatment of acute bacterial maxillary sinusitis. Sparfloxacin Multicenter AMS Study Group. Clin Ther 1999;21:340-52.

27. Low DE, Desrosiers M, McSherry J, et al. A practical guide for the diagnosis and treatment of acute sinusitis. CMAJ 1997;156(Suppl 6):S1-14. 
28. Hickner JM, Bartlett JG, Besser RE, et al. Principles of appropriate antibiotic use for acute rhinosinusitis in adults: background. Ann Intern Med 2001;132(6):498-505.

29. Ah-See K. Sinusitus (acute). Clin Evid (Online) 2004;11:129-30.

30. Vincent MT, Celestin N, Hussain AN. Pharyngitis. Am Fam Physician 2004;69: 1465-70.

31. Mclsaac WJ, Goel V, To T. The validity of a sore throat score in family practice. CMAJ 2000;163:811-5.

32. Mayes T, Pichichero ME. Are follow-up throat cultures necessary when rapid antigen detection tests are negative for group A streptococci? Clin Pediatr (Phila) 2001;40:191-5.

33. Bisno AL. Acute pharyngitis. N Engl J Med 2001;344:204-11.

34. Evans A, Niederman J. Epstein-Barr virus. Viral infections of human epidemiology and control. New York: Plenum Publishing; 1989. p. 265.

35. Rea TD, Russo JE, Katon W, et al. Prospective study of the natural history of infectious mononucleosis caused by Epstein-Barr virus. J Am Board Fam Pract 2001; 13:234-42.

36. Aldrete JS. Spontaneous rupture of the spleen in patients with infectious mononucleosis. Mayo Clin Proc 1992;67:910-2.

37. File TM. Community-acquired pneumonia. Lancet 2003;362:1991-2001.

38. Mandell LA, Bartlett JG, Dowell SF, et al. Infectious Diseases Society of America. Update of practice guidelines for the management of community-acquired pneumonia in immunocompetent adults. Clin Infect Dis 2003;37:1405-33.

39. Gonzales R, Sande MA. Uncomplicated acute bronchitis. Ann Intern Med 2000; 133:981-91.

40. Blinkhorn RJ Jr. Upper respiratory tract infections. In: Baum GL, editor. Textbook of pulmonary diseases. 6th edition. Philadelphia: Lippincott-Raven; 1998. p. 493-502.

41. Irwin RS, Curley FJ, Bennett FM. Appropriate use of antitussives and protussives. A practical review. Drugs 1993;46:80-91.

42. Hueston WJ. Albuterol delivered by metered-dose inhaler to treat acute bronchitis. J Fam Pract 1994;39:437-40.

43. Tingle LE, Molina D, Calvert CW. Acute pericarditis. Am Fam Physician 2007; 76(10):1509-14.

44. Spodick DH. Acute pericarditis: current concepts and practice. JAMA 2003;289: 1150-3.

45. Spodick DH. Pericardial diseases. In: Braunwald E, Zipes DP, Libby P, editors. Heart disease, a textbook of cardiovascular medicine. 6th edition. Philadelphia: WB Saunders; 2001. p. 1823-76.

46. Maisch B, Seferovic PM, Ristic AD, et al. for the Task Force on the Diagnosis and Management of Pericardial Diseases of the European Society of Cardiology. Guidelines on the diagnosis and management of pericardial diseases executive summary. Eur Heart J 2004;25:587-610.

47. Wynne J, Braunwald E. The cardiomyopathies and myocarditides. In: Braunwald E, Fauci A, Kasper D, editors. Harrison's principles of internal medicine. 15th edition. New York: McGraw Hill; 2001. p. 1359-65.

48. Puranic R, Chow CK, Duflou JA. Sudden death in the young. Heart Rhythm 2005; 2(12):1277-82.

49. Durakovic Z, Durakovic M, Skavic J. Myopericarditis and sudden cardiac death due to physical exercise in male athletes. Coll Antropol 2008;32(2):399-401.

50. Martin AG, Koboyashi GS. Superficial fungal infection: dermatophytosis, tinea nigra, piedra. In: Freedberg IM, et al, editors. 5th edition, Fitzpatrick's dermatology in general medicine, vol. 2. New York: McGraw-Hill; 1999. p. 2337-57. 
51. Adams BB. Tinea corporis gladitorum. J Am Acad Dermatol 2002;47(2): 286-90.

52. Hainer BL. Dermatophyte infections. Am Fam Physician 2003;67:101-8.

53. Cobbold RJ, Macdonald A. Molluscum contagiosum as a sexually transmitted disease. Practitioner 1970;204:416-9.

54. Schwartz JJ, Myskowski PL. Molluscum contagiosum in patients with human immunodeficiency virus infection. A review of twenty-seven patients. J Am Acad Dermatol 1992;27:583-8.

55. Buller RM, Plumbo GJ. Poxvirus pathogenesis. Microbiol Rev 1991;55:80-122.

56. Ashcraft KA, Hunzeker J, Bonneau RH. Psychological stress impairs the local CD8+ T cell response to mucosal HSV-1 infection and allows for increased pathogenicity via a glucocorticoid receptor mediated mechanism. Psychoneuroendocrinology 2008;33(7):951-63 Epub 2008 Jul 25.

57. Brian B. Adams viral skin infections. In: Adams BB, editor. Sports dermatology. Springer; 2006. p. 35-46.

58. Nerukar LS, West F, May M, et al. Survival of herpes simplex virus in water specimens collected from hot tubs in spa facilities and on plastic surfaces. JAMA 1983;250:3081-3.

59. Ichihashi M, Nagai H, Matsunaga K. Sunlight is an important causative factor of recurrent herpes simplex. Cutis 2004;74(Suppl 5):14-8.

60. Holland EJ, Nahanti RL, Belongia EA, et al. Ocular involvement in an outbreak of herpes gladiatorum. Am J Ophthalmol 1992;114:680-4.

61. White WB, Grant-Kels JM. Transmission of herpes simplex virus type 1 infection in rugby players. JAMA 1984;252:533-5.

62. Salgado CD, Farr BM, Calfee DP. Community acquired methicillin-resistant Staphylococcus aureus: a meta-analysis of prevalence and risk factors. Clin Infect Dis 2003;36:131-9.

63. Brian B. Adams bacterial skin infections. In: Adams BB, editor. Sports dermatology. Springer; 2006. p. 3-34.

64. Nessel L. Florida fighter learns of MRSA infection after MMA bout. Florida Today February 21, 2009.

65. Kazakova SV, Hagemen JC, Matava M, et al. A clone of methicillin-resistant Staphylococcus aureus among professional football players. N Engl J Med 2005;352(5):468-75.

66. Bartlett PC, Martin RJ, Cahill BR. Furunculosis in a high school football team. Am J Sports Med 1982;10:371-4.

67. Sedgewick PE, Dexter WW, Smith CT. Bacterial dermatosis in sports. Clin Sports Med 2007;26:383-96.

68. Alcena V. Boxing and the transmission of HIV. N Y State J Med 1988;88(7):392.

69. Drotman DP. Professional boxing, bleeding and HIV testing. JAMA 1996;276(3): 193.

70. Centers for Disease Control and Prevention, national prevention information network. Available at: www.cdc.gov/hiv/resources. Accessed February 3, 2009.

71. Nattiv A, Puffer JC. Lifestyles and health risks of collegiate athletes. J Fam Pract 1991;33:585-90.

72. Sklarek HM, Mantovani RP, Erens E, et al. AIDS in a bodybuilder using anabolic steroids. N Engl J Med 1984;311:1701.

73. Torre D, Sampietro C, Ferraro G, et al. Transmission of HIV-1 infection via sports injury. Lancet 1990;335:1105.

74. O'Farrell N, Tovey SJ, Morgan-Capner P. Transmission of HIV-1 infection after a fight. Lancet 1992;339:246. 
75. Ippolito G, Del Poggio P, Arici C. Transmission of zidovudine-resistant HIV during a bloody fight. JAMA 1992;272:433-4.

76. Perlmutter BL, Glaser JB, Oyugi SO. How to recognize and treat acute HIV syndrome. Am Fam Physician 1999;60:535-46.

77. Jordan BD. Aids and boxing. In: Jordan B, editor. Medical aspects of boxing. Boca Raton (FL): CRC Press; 2000. p. 317-22.

78. Souza PM, Jacob-Filho W, Santarém JM, et al. Progressive resistance training in elderly HIV positive patients: does it work? Clinics (Sau Paulo) 2008;63(5): 619-24.

79. Domingo hit with hepatitis B, Rocha fight called off. Available at: Boxingscene. com. Accessed November 21, 2008.

80. Bunce S. Boxing: skelton has the bulk and skill to shock Chagaev. Available at: independentNewsandMedia.com. Accessed January 19, 2008.

81. Lin KW, Kirchner JT. Hepatitis B. Am Fam Physician 2004;69:76.

82. Pediatrics Committee on Sports Medicine and Fitness. Human immunodeficiency virus and other blood-borne viral pathogens in the athletic setting. Pediatrics 1999;104:1400-3.

83. Parana R, Lyra L, Trepo C. Intravenous vitamin complexes used in sporting activities and transmission of HCV in Brazil. Am J Gastroenterol 1999;94:857-8. 